\title{
UNIQUENESS THEOREM IN COMPLETE RESIDUATED ALMOST DISTRIBUTIVE LATTICES
}

\author{
G.C. RAO AND S.S. RAJU \\ Department of Mathematics \\ Andhra University \\ Visakhpatanam - 530003, A.P., India \\ e-mail: gcraomaths@yahoo.co.in \\ ssrajumaths@gmail.com
}

\begin{abstract}
Important properties of primary elements in a complete residuated ADL $L$ and the uniqueness theorem in a complete complemented residuated ADL $L$ are proved.

Keywords: Almost Distributive Lattice (ADL), residuation, multiplication, residuated ADL, complete residuated ADL, primary decomposition, reduced primary decomposition and normal primary decomposition.
\end{abstract}

2010 Mathematics Subject Classification: 06D99, 06D15.

\section{REFERENCES}

[1] R.P. Dilworth, Abstract residuation over lattices, Bull. Amer. Math. Soc. 44 (1938) 262-268. doi:10.1090/S0002-9904-1938-06736-5

[2] G.C. Rao, Almost Distributive Lattices, Doctoral Thesis (Department of Mathematics, Andhra University, Visakhapatnam, 1980).

[3] G.C. Rao and S.S. Raju, Complete residuated Almost Distributive Lattices, Communicated to the Journal Discussiones Mathematicae-General Algebra and Applications.

[4] G.C. Rao and S.S Raju, Decomposition theorems in principal residuated Almost Distributive Lattices, Communicated to the Journal The Journal of the Chung Cheong Mathematical Society.

[5] G.C. Rao and S.S Raju, Noether Almost Distributive Lattices, Bull. Intrnat. Math. Virtual Institute 9 (2019) 39-46. 
[6] G.C. Rao and S.S Raju, Principal Residuated Almost Distributive Lattices, Bull. Internat. Math. Virtual Institute 9 (2019) 219-230.

[7] G.C. Rao and S.S Raju, Residuated Almost Distributive Lattices - I, Internat. J. Math. Archive 8 (2017) 143-152.

[8] G.C. Rao and S.S Raju, Residuated Almost Distributive Lattices - II, Internat. J. Math. Archive 8 (2017) 190-198.

[9] G.C. Rao and Venugopalam Undurthi, Complete Almost Distributive Lattices, Asian-European J. Math. 7 (2014) 1450052 (8 pages).

doi:10.1142/S1793557114500521

[10] U.M. Swamy and G.C. Rao, Almost Distributive Lattices, J. Aust. Math. Soc. (Series A) 31 (1981) 77-91. doi:10.1017/S1446788700018498

[11] M. Ward and R.P. Dilworth, Residuated lattices, Proceedings of the National Academy of Sciences 24 (1938) 162-164. doi:10.1073/pnas.24.3.162

[12] M. Ward and R.P. Dilworth, Residuated lattices, Trans. Amer. Math. Soc. 45 (1939) $335-354$. doi:10.1090/S0002-9947-1939-1501995-3

[13] M. Ward, Residuated distributive lattices, Duke Math. J. 6 (1940) 641-651. doi:10.1215/S0012-7094-40-00649-4

Received 29 November 2018

Revised 11 July 2019

Accepted 12 July 2019 